\title{
Comercio intraindustrial de Santander con los países de la Alianza del Pacífico
}

\section{Intraindustry trade of Santander with Pacific Alliance's countries}

\author{
Edgar Javier Gómez Parada \\ Leonardo Javier Caraballo ${ }^{2}$ \\ Leidy Dayanna Peña Hernández ${ }^{3}$
}

\begin{abstract}
Resumen
El comercio intraindustrial es el flujo comercial en doble vía entre dos regiones dentro de una misma actividad industrial; identifica la complementariedad y competencia entre dos ofertas exportables. El trabajo determina el nivel del comercio intraindustrial del departamento de Santander, Colombia, con los países de la Alianza del Pacífico; para ello se hace el cálculo del índice de Grubel y Lloyd con una desagregación a 4 dígitos para partidas arancelarias entre los años 2013 - 2018 y se hace una valoración del nivel de dicho intercambio. Se incluyeron 112 partidas arancelarias consideradas relevantes por el valor exportado. Se encuentra comercio intraindustrial de Santander con los países de la Alianza en 22,3\% de dichas partidas. Solo se presenta alta relevancia en una partida y relevancia media en 3, en ambos casos con México; en las demás partidas arancelarias donde se presenta comercio en doble vía el nivel es bajo.
\end{abstract}

\section{Palabras clave}

Comercio intraindustrial, Comercio intraindustrial de Santander, Índice de Grubel y Lloyd, Alianza del Pacífico.

\section{Códigos de clasificación JEL: F14, F15, F20, 054.}

\begin{abstract}
Intraindustry trade is the two-way trade flow between two regions within the same industrial activity. Intratraindustry trade serves to identify the complementarity and competition between two exportable offers. To measure of the level of intrindustry trade of department of Santander, Colombia with Alliance Pacific's countries, Grubel y Lloyd index was used. It is calculated with information about exports and imports of each country at 4-digit code or heading between 2013-2018 and based on the value of the index, a categorization is used to assess level of significance of intraindustry trade of each heading. In this study were included 112 heading considered relevant for the exported value. Intraindustry trade of Santander with the Alliance Pacific's countries is found in $22,3 \%$ of these items. IGL reflects a high relevance in a heading, medium relevance in 3 , in both items with Mexico. In the other headings there are low relevance of intraindustry trade.
\end{abstract}

\section{Keywords}

Intraindustry trade, Grubel y Lloyd index, Intraindustry trade of Santander, The Pacific Alliance.

1 Universidad Santo Tomás Bucaramanga. Correo electrónico: edgar.gomez01@ustabuca.edu.co Código ORCID: 0000-0003-0864-3740

2 Universidad Santo Tomás Bucaramanga. Correo electrónico: leonardo.caraballo@ustabuca.edu.co Código ORCID: 0000-0002-8350-3556

3 Universidad Santo Tomás Bucaramanga. Correo electrónico: leidy.pena@ustabuca.edu.co Código ORCID: 0000-0001-9600-7976 


\section{Introducción}

La Alianza del Pacífico surge como una iniciativa económica entre Chile, Colombia, México y Perú, que se enmarca en un regionalismo abierto y profundo. Hernández y Muñoz (2015) destacan que es abierto por el nivel de apertura comercial de cada uno de los miembros y por las características propias del acuerdo de integración que liberan los mercados y el comercio de bienes y servicios, además de regirse por normas establecidas por la Organización Mundial del Comercio (OMC). Mientras que la tipología de integración profunda es determinada por los propósitos de la Alianza que tienen alcances multidimensionales con procesos de supranacionalidad.

El comercio intrarregional es aquel que se presenta entre dos economías que corresponden a una región de una circunscripción geográfica dada por su proximidad o una región definida a través de acuerdos de integración económica. En el comercio intrarregional puede presentarse comercio intraindustrial e interindustrial; el primero se refiere a importaciones y exportaciones entre actividades industriales similares y, el segundo, a actividades industriales completamente diferentes (Aghlmand, Rahimi, Farrokh, Nabilou y Yusefzadeh, 2018).

El estudio del comercio intraindustrial dentro de las nuevas teorías del comercio internacional trata de explicar el hecho de las exportaciones e importaciones que realizan los países dentro de una misma actividad económica. Según Brakman, Garretsen y Van Marrewijk (2009), el comercio intraindustrial se puede dar en dos sentidos, horizontal cuando se realiza entre industrias similares y con el mismo nivel de procesamiento o intensidad tecnológica y, vertical, cuando está dentro de la misma actividad industrial, pero con distinto nivel de procesamiento. Sotomayor (2009) explica que las diferencias en sentido vertical y horizontal pueden deberse principalmente a aspectos tecnológicos y de calidad.

El objetivo del estudio se orientó en determinar el nivel del comercio intraindustrial del departamento de Santander, Colombia con los países de la Alianza del Pacífico para la identificación de alternativas de alianzas y encadenamientos productivos que conlleven al establecimiento de cadenas regionales de valor en uno de los propósitos de la Alianza, que es buscar inserción en los mercados asiáticos de manera conjunta. La conformación de dichas cadenas regionales de valor es más factible en la medida en que se presenten intercambios comerciales intraindustriales.

Para un departamento como Santander, que busca mayor proyección de sus exportaciones, es importante hacer una mirada a los países de Chile, México y Perú como opción de alianzas productivas que permitan robustecer capacidades de internacionalización de sus sectores productivos. Estos mercados en su conjunto representaron en el 2018 aproximadamente el 14,3\% del total exportado por el departamento de Santander. 
Para la determinación del nivel de comercio intraindustrial se seleccionó el índice de Grubel y Lloyd, por presentar facilidad de cálculo y de acceso a los datos, ya que se hace con los valores de las exportaciones e importaciones; y para la interpretación del nivel de comercio intraindustrial se establece un rango entre bajo, medio y alto de acuerdo con el valor del índice, siguiendo la propuesta de Caicedo y Mora (2011). Se toma como rango del estudio desde el 2013 hasta el 2018.

Entre los principales resultados del estudio se evidencia la importancia que han adquirido los países de la Alianza del Pacífico en las exportaciones de Santander entre los años 2013 - 2018 y un nivel de comercio intraindustrial predominantemente bajo entre este departamento con cada uno de los demás países integrantes de la Alianza.

\section{Definición del comercio intraindustrial}

Desde el auge en la apertura comercial a nivel mundial, los países han buscado estrategias para su crecimiento económico a través de la competitividad, lo cual era explicado en las teorías clásicas del comercio con Adam Smith (Villalobos y Quiroga, 2017) y David Ricardo (Kalim, 2018). No obstante, a partir de 1960, algunos estudiosos de la materia encontraron que el comercio internacional había evolucionado a otros ámbitos y las teorías clásicas ya no abarcaban dicho fenómeno.

Estudios sugieren que las economías a nivel internacional pueden tener dos tipos de comercio según la industria, el interindustrial y el intraindustrial. El primer tipo hace referencia a los flujos comerciales entre países en industrias diferentes, lo cual corresponde a la ventaja comparativa que fundamenta David Ricardo. El segundo tipo son las exportaciones e importaciones que tiene un país dentro de la misma industria (Duregger, 2019). A este último, Sotomayor (2008) lo denomina como comercio coincidente o trade overlap entendiéndolo como el resultado de las variaciones en los requerimientos de los factores productivos dentro de una misma industria (comercio intraindustrial), más que entre ellas (comercio interindustrial).

Pick, Henderson, Kinsey y Sheldon (2018) destacan las clasificaciones del comercio intraindustrial de acuerdo con la diferenciación de productos basados en las economías de escala, los bienes funcionalmente homogéneos y la brecha tecnológica. Frente al tema, Lucangeli (2007) lo distingue así: a) los bienes que siendo fabricados con insumos diferentes pueden sustituirse en el consumo, b) aquellos que en su producción son homogéneos, pero en el uso no son sustitutos y c) los productos que en su funcionalidad son similares, pero se diferencian con atributos de diseño, estilo, calidad o marca.

Con respecto a la diferenciación de productos este puede presentarse de tipo horizontal o vertical (Aziz, Hossain y Mowlah, 2018); el modelo de diferenciación horizontal de producto es definido por Montaner y Orts (1996) como los bienes que 
son producidos de forma idéntica, pero son ofrecidos por cada empresa con un factor diferenciador, creando en el mercado una estructura de competencia monopolística. Por su parte Moreno y Posada (2007) explican el comercio intraindustrial de tipo horizontal por medio del esquema de demanda, el cual se caracteriza por la diversificación del consumo y el aumento en la variedad de productos.

De igual forma, el comercio intraindustrial vertical se entiende con la similitud de preferencias que sintetiza el comportamiento de las exportaciones e importaciones en coherencia con la situación de la demanda. Es decir, que "se producen y exportan calidades de un mismo bien demandadas por la mayoría y se importan aquellas demandadas por la minoría" (Moreno y Posada, 2006, p. 4).

Paralelamente, León y Dussel (2001) consideran una diferenciación intraindustrial de forma vertical cuando una misma cadena de producción se localiza en diferentes países, dando lugar a la reexportación de bienes y de forma horizontal cuando dos cadenas de producción independientes llevan al intercambio internacional de bienes de la misma industria con un mismo nivel de elaboración, argumentado por López y Rodil (2008).

Dado lo anterior, es necesario considerar el comercio intrafirma entendido como aquel que se presenta en el interior de las empresas que están bajo la misma estructura organizacional y el cual también se categoriza en: a) vertical, cuando los flujos comerciales corresponden a partes y componentes en el encadenamiento productivo y comercial del bien y b) horizontal, en donde el comercio se presenta entre empresas de distintas ramas industriales con productos diferentes (Moreno y Posada, 2006).

En este contexto, la integración internacional se hace fundamental para el fortalecimiento del ecosistema productivo de las economías en relación con la globalización. Por medio de la integración regional los países pueden relocalizar las etapas productivas con las estructuras del acuerdo comercial en materia arancelaria; conjuntamente, se abre la opción de inserción a las cadenas globales y regionales de valor en la integración productiva de tipo vertical y horizontal (Molinari, Bembi y de Ángeles, 2012) (Baccini, Dur y Elsig, 2018).

\section{Revisión bibliográfica}

De acuerdo con Arza y Almanza (2014), Balassa fue un precursor del concepto de comercio intraindustrial, estudiando el caso estadounidense con respecto a 38 socios comerciales para la época de 1979 con el resultado de que a mayor similitud de economías mayor interacción comercial de tipo intraindustrial tendrán.

Para el contexto regional sudamericano durante el 2003-2011 se encontró que Colombia mantuvo sus índices de comercio intraindustrial, mientras que todos los demás países -salvo Guyana, Bolivia y Venezuela- crecieron en este aspecto, destacando que sus índices son superiores en la región con industrias del sector primario, 
así como las manufacturas de contenido tecnológico medio, mientras que en la relación con el resto del mundo predominan las exportaciones de recursos naturales y las importaciones de productos industriales (Gaya y Michalczewsky, 2014).

De acuerdo con Lucangeli (2007), los flujos comerciales intraindustriales del Mercosur podrían explicarse por la presencia de empresas multinacionales que realizan sus operaciones bajo el modelo de comercio intrafirma, aprovechando las ventajas de cada país para dividir las tareas por realizar conforme a la movilidad del trabajo, los salarios y los factores de producción que permitan obtener beneficios de bajos costos. En este sentido, el comercio intra-Mercosur tiene el potencial de contribuir al fortalecimiento de cadenas productivas regionales (Molinari, Bembi y de Ángeles, 2012).

Desde 1992 hasta el 2004 el comercio intraindustrial para productos químicos entre Brasil y Argentina ha tenido un incremento porcentual del 30\%, alcanzando un índice de 75,9 en el 2004, lo cual puede entenderse desde la especialización de las economías con factores tecnológicos (Lucangeli, 2007).

Sin embargo, Molinari, Bembi y de Ángeles (2012) fundamentan que el comercio intraindustrial entre los dos países es horizontal para bienes intermedios, mientras que cuando se comercializan productos finales corresponden mayoritariamente a intercambios intraindustriales de tipo vertical.

En la relación comercial entre Colombia y Estados Unidos se analizan los productos industriales considerados en el BACI donde solo el $9 \%$ presentan comercio de doble vía, de los cuales el $68 \%$ obtuvieron un índice de comercio intraindustrial inferior a 25, de acuerdo con la fórmula aplicada de Grubel y Lloyd se interpreta como bajo según las categorías establecidas por los autores para efectos de análisis del estudio (superior a 50 corresponde a un índice alto, entre 25 y 50 es considerado medio e inferior a 25 es bajo), sintetizando que los productos comercializados entre Colombia y Estados Unidos son en su mayoría de tipo interindustrial (Caicedo y Mora, 2011).

Durante 1991-2015 Colombia y Estados Unidos presentan comercio intraindustrial vertical en la cría de caballos y la elaboración de abono, aunque su significancia es baja, esto de acuerdo con la metodología utilizada de comercio intraindustrial marginal de Brulhart (Jiménez, 2018).

Desde la consideración de los determinantes del comercio intraindustrial Arza y Almanza (2014); para el periodo 2000-2011, en el caso de Colombia en relación con sus principales socios comerciales, encuentran que de las nueva variables consideradas, cuatro tienen una relación negativa con respecto al índice de Grubel y Lloyd, las cuales son las variables que caracterizan al país en cuanto a la dotación de factores, la distancia, la balanza comercial y la relación capital trabajo; mientras que la estructura del mercado, la orientación comercial, el lenguaje, la industria y la diferenciación vertical de productos generan un efecto positivo con el índice de comercio intraindustrial. 
En el mismo sentido, Moscoso y Vásquez (2005) analizan los determinantes del comercio intraindustrial en el grupo de los tres (G3) Colombia, Venezuela y México; argumentan que el comercio intraindustrial disminuye la dependencia de las exportaciones en los productos primarios, conduce a procesos de integración regional productiva y posibilita encadenamientos productivos que conduzcan a la reducción de costos. Encuentra que los flujos de comercio intraindustrial entre estos tres países no es tan significativo como en los países desarrollados, y que aumentar dicho tipo de comercio se requiere de la diversificación de la oferta exportable. Entre los factores que influyeron en el comercio intraindustrial en el G3 estuvieron la reducción tarifaria y la crisis mexicana en el año 1994, pero principalmente la inversión en investigación y desarrollo que originó productos exportables con mayor contenido tecnológico.

Teniendo en cuenta la participación de Colombia en la Comunidad Andina se analiza el comportamiento de los flujos comerciales intrarregionales, con el fin de identificar el comercio intraindustrial dentro de la CAN, para lo cual se usa la medición de Grubel y Lloyd, solucionando los problemas de agregación y de sesgo geográfico. Con los resultados se determina un índice de $13,5 \%$ en promedio para 1992-2004 entre Colombia y la CAN con productos de industrialización media. No obstante, los países con mayor factorización intraindustrial son Ecuador y Venezuela, siendo principalmente de carácter vertical con el mayor porcentaje de alta calidad para Venezuela (Moreno y Posada, 2007).

Pasando a la Alianza del Pacífico, Hernández y Muñoz (2015) estudian el comercio intrarregional a nivel de exportaciones, importaciones y ventajas comparativas reveladas, hallando que las exportaciones intra-alianza no superaron el $4 \%$ de las exportaciones totales al mundo en el 2000-2012, señalando que los países miembros cuentan con economías similares con respecto a la oferta exportable, dominando principalmente aquellos relacionados con el sector primario de agropecuaria y minería, aunque de esta similitud se puede producir encadenamientos productivos que como bloque económico puedan aprovechar para fortalecer su competitividad llegando a los mercados asiáticos.

\section{Metodología}

Con el fin de determinar el comercio intraindustrial entre el departamento de Santander, con respecto a los países miembros de la Alianza del Pacífico, se toman las exportaciones e importaciones a nivel de partidas arancelarias a cuatro dígitos entre los años 2013-2018, para minimizar niveles de solapamiento entre los flujos de las actividades industriales. Los datos se obtienen de la base de datos Wisertrade que proporciona información sobre exportaciones e importaciones; los datos suministrados por dicha base, para el caso de Colombia, provienen del Departamento Nacional de Estadísticas - DANE. Se hace una normalización de datos estableciendo criterios 
de inclusión y exclusión de partidas arancelarias; se hace el cálculo del índice de Grubel y Lloyd para el flujo comercial de Santander con cada uno de los demás países de la Alianza del Pacífico.

\section{Normalización de los datos}

Se incluyen aquellas partidas arancelarias que presentan flujo comercial para las exportaciones e importaciones durante el periodo 2013-2018 en al menos dos años, y aquellas partidas arancelarias cuyo valor sea superior a los 3000 USD en al menos un año para las exportaciones de Santander. Para ser considerado comercio intraindustrial se incluyen aquellas partidas en las que se presenta comercio en doble vía en el mismo año.

Para el flujo comercial de Santander con Chile, al normalizar los datos en el periodo de 2013 al 2018 con exportaciones en al menos 2 años quedaron 34 partidas arancelarias, de ellas 28 presentaron exportaciones por encima de los 3000 USD al menos en un año. De estas solo se presenta comercio en doble vía en el mismo periodo de tiempo en 5 partidas.

En el caso de Santander con México de 35 partidas incluidas en los criterios de inclusión, se encontró comercio en doble vía en 15 posiciones arancelarias. En las exportaciones del departamento de Santander hacia Perú se incluyeron 43 partidas, y en 5 se presenta comercio en doble vía.

\section{Cálculo del índice de comercio intraindustrial}

Se aplica el índice de Grubel y Lloyd, cuyo resultado oscila entra 0 y 1, siendo nulo el comercio intraindustrial cuando esté en 0 y siendo completamente intraindustrial cuando esté en 1.

$$
I G L_{i t}^{k}=1-\frac{\left|X_{i j t}^{k}-M_{i j t}^{k}\right|}{X_{i j t}^{k}+M_{i j t}^{k}} \quad[1]
$$

Donde:

IGL $=$ Índice de Grubel y Lloyd

$\mathrm{k}=$ Partida arancelaria a 4 dígitos

$\mathrm{i}=$ Región: departamento Santander

$\mathrm{j}=$ Mercado país miembro de la Alianza del Pacífico

$\mathrm{t}=\mathrm{A} \tilde{\mathrm{no}}$

$X_{i j t}^{k}$ : Exportaciones de Santander al país j en la partida k en al año t.

$M_{i j t}^{k}$ : Importaciones de Santander del país j en la partida k en el año t. 


\section{Valoración del índice Grubel y Lloyd}

Para medir la significancia del comercio intraindustrial se establece una valoración, siguiendo la propuesta de Caicedo y Mora (2011), la cual establece que el nivel de comercio intraindustrial es bajo cuando el índice de Grubel y Lloyd es inferior a 0,25 , presenta nivel medio cuando el valor se encuentra entre 0,25 y 0,50 y es alto cuando el índice es superior a 0,50 .

Tabla 1. Valoración del indice de Grubel y Lloyd

\begin{tabular}{cc}
\hline Indicador & Valoración \\
\hline Superior a 0,50 & Alto \\
\hline Entre 0,25 y 0,50 & Medio \\
\hline Inferior 0,25 & Bajo \\
\hline
\end{tabular}

Fuente: Elaboración propia.

Para la valoración, se calcula el promedio del valor del índice de Grubel y Lloyd entre los años 2013-2018 para cada partida arancelaria.

\section{Resultados}

\section{Exportaciones de Santander a la Alianza del Pacífico}

Las exportaciones totales de Santander reportaron decrecimiento del 19,32\% entre los años 2013-2018, mientras que las exportaciones que Santander dirige a los mercados de la Alianza del Pacífico, Chile, Perú y México, presentaron crecimiento del 30,49\% en el mismo periodo; esto indica que los países de la Alianza han adquirido importancia relativa en las exportaciones del Departamento.

Aunque se presentan similitudes culturales, sociales, económicas y políticas entre los cuatro países de la Alianza del Pacífico; la participación de las exportaciones del departamento de Santander a los mercados de Chile, México y Perú fueron bajas entre los años 2013-2018 (4,75\%; 5,59\% y 3,96\%, respectivamente en el año 2018) y representaron el $14,31 \%$ del total exportado en dicho año. Es de anotar que el principal destino de las exportaciones del departamento es Estados Unidos (con participación del 25,7\% en el 2018) seguido por España (con participación del 12,1\% aproximadamente en el 2018).

En total, la Alianza del Pacífico como socio comercial de Santander aumentó $61,83 \%$ desde el 2013 hasta el 2018, al pasar de 8,85\% de participación en exportaciones santandereanas en 2013 al 14,31\% para el 2018 . 


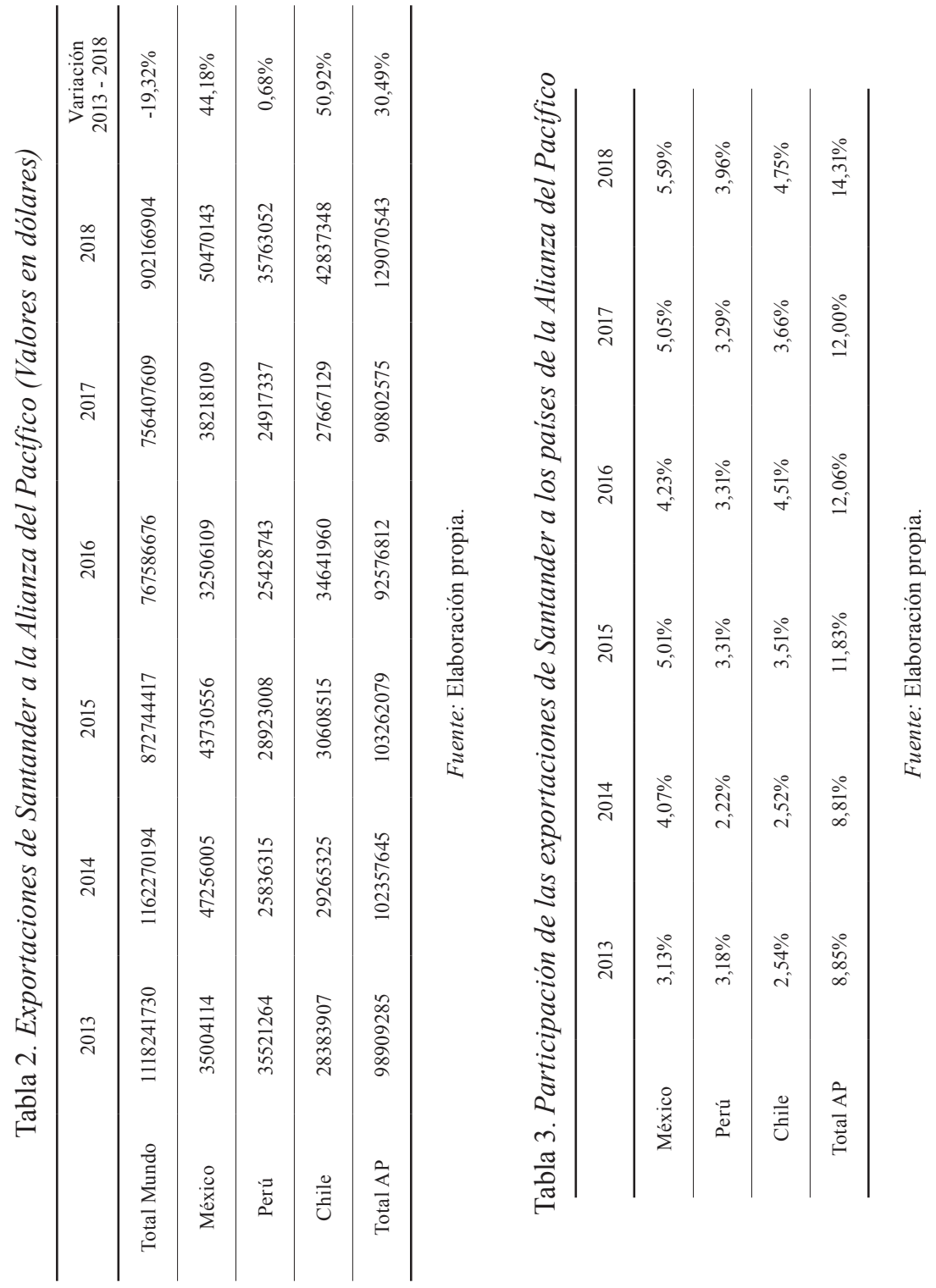




\section{Índice de Grubel y Lloyd entre Santander y la Alianza del Pacífico}

En términos generales se observa nivel de comercio intraindustrial bajo entre los flujos comerciales de Santander con Chile y Perú y medio de Santander con México; los valores del índice de Grubel y Lloyd para el caso de Chile y Perú no superan el 0,1; hecho que refleja que en muy pocos productos se presenta comercio intraindustrial (tabla 4).

\section{Índice de Grubel y Lloyd en la relación Santander - Chile}

De 34 partidas arancelarias donde se presentaron exportaciones por encima de 3000 dólares de Santander a Chile entre 2013-2018, tan solo en 5 de ellas se encontró comercio en doble vía; es decir, que en ellas se evidenció comercio intraindustrial en al menos un año.

Desde el 2013 hasta el 2016 predominó el comercio intraindustrial bajo en las 5 partidas; no obstante, en el 2014 se presentó nivel alto para la partida 3901, y nivel medio para la partida 8421 en el 2015 y 8544 en el 2017. Durante el año 2018 no se presentó comercio intraindustrial entre Santander y Chile.

\section{Índice de Grubel y Lloyd en la relación Santander - México}

Durante el 2013-2018 en las exportaciones de Santander a México, según los criterios de normalización de datos, se incluyeron 35 partidas arancelarias, en las cuales se presentó comercio intraindustrial en 15 de ellas.

En el comercio intraindustrial del departamento de Santander con México es destacable que en todos los años de estudio se presentó nivel alto al menos en una partida arancelaria; en el 2013 en la partida 8421 con un índice de 0,9778, para el 2014 la partida 8422 con 0,6343 , destacando que ambas pertenecen al capítulo 84 de máquinas, aparatos y artefactos mecánicos; en el 2015 y 2018 se presentó comercio intraindustrial alto en dos partidas arancelarias, mientras que en 2016 y 2017 se destacaron tres en cada año.

La partida arancelaria con mayor nivel de comercio intraindustrial en el promedio de los cinco años fue la 8421 "Centrifugadoras, incluidas las secadoras centrífugas; aparatos para filtrar o depurar líquidos o gases”, que presenta índice alto, aunque con niveles bajos en el 2014 con 0,0129 y en 2018 con 0,2304.

Cabe señalar que la partida 8419 presentó nivel alto en el año 2016 con un índice de 0,5655 , sin embargo, para los demás años de estudio el comercio intraindustrial fue nulo entre Santander y México.

Por otra parte, la partida 8436 , presentó nivel de comercio intraindustial medio entre Santander y México en casi todos los años, con una dinámica descendente, ya que en 2014 el índice fue de 0,4802, en el 2016 fue de 0,3950 y en 2017 de 0,3251, mientras para el 2018 disminuyó a nivel bajo con 0,1761 . 
Desde el punto de vista anual, el año con mayor cantidad de partidas arancelarias con comercio intraindustrial de nivel medio fue el 2014 con 5 partidas, en tanto que para el 2013 y 2018 solo hubo una partida arancelaria en cada año.

El índice de Grubel y Lloyd para Santander con respecto a México para las 15 partidas arancelarias que presentaron flujos comerciales en doble vía desde el 2013 hasta el 2018 evidencia dominancia de comercio intraindustrial con nivel bajo.

\section{Índice de Grubel y Lloyd en la relación Santander - Perú}

Para el análisis del comercio intraindustrial del departamento Santander con respecto a Perú, al hacer los procesos de inclusión quedaron 43 partidas arancelarias en donde Santander exportó a Perú montos superiores a \$3000 USD, al menos en un año entre el 2013-2018. Se encontraron cinco partidas arancelarias comercializadas en doble vía.

Es de resaltar que solo se presenta un índice de comercio intraindustrial medio para el año 2015 correspondiente a la partida arancelaria 6005 "tejidos de punto por urdimbre", mientras que los demás resultados revelan un comercio intraindustrial bajo entre Santander y Perú, destacando que para la partida 3923 "artículos para el transporte o envasado de plástico" se obtiene comercio intraindustrial en los 6 años de estudio.

Al comparar las características del comercio intraindustrial de Santander con cada uno de los países de la Alianza del Pacífico con base en el índice Grubel y Lloyd entre los años 2013-2018 se encuentra que solo en la partida 8421 se presentó comercio intraindustrial del departamento con los tres países, con nivel alto para el caso de México y bajo con Chile y Perú. En la partida 7318 se encuentra comercio intraindustrial de Santander con Chile y Perú, en ambos casos con nivel bajo y en la partida 3923 con México y Perú, también con niveles bajos.

Según Hernández y Muñoz (2015) el hecho de que el comercio intraindustrial entre los países de la Alianza del Pacífico sea bajo, se debe, por una parte, a que sus economías no son complementarias y más bien se presenta rivalidad y, por otra, a que las exportaciones de cada país tienen alta concentración en el caso de Colombia y México a Estados Unidos y de Chile y Perú a China y a países asiáticos.

Dada la situación anterior, Márquez (2015) enfatiza en la necesidad de buscar estrategias para incrementar el comercio intrarregional entre los miembros de la AP, ya que la tendencia es a la disminución del comercio entre sí de estos países, aun a pesar de que existen mecanismos para liberalizar la circulación de mercancías. Aunque para el caso del departamento de Santander la tendencia entre los años 2013-2018 fue al crecimiento de las exportaciones a cada uno de los mercados de la Alianza del Pacífico. 

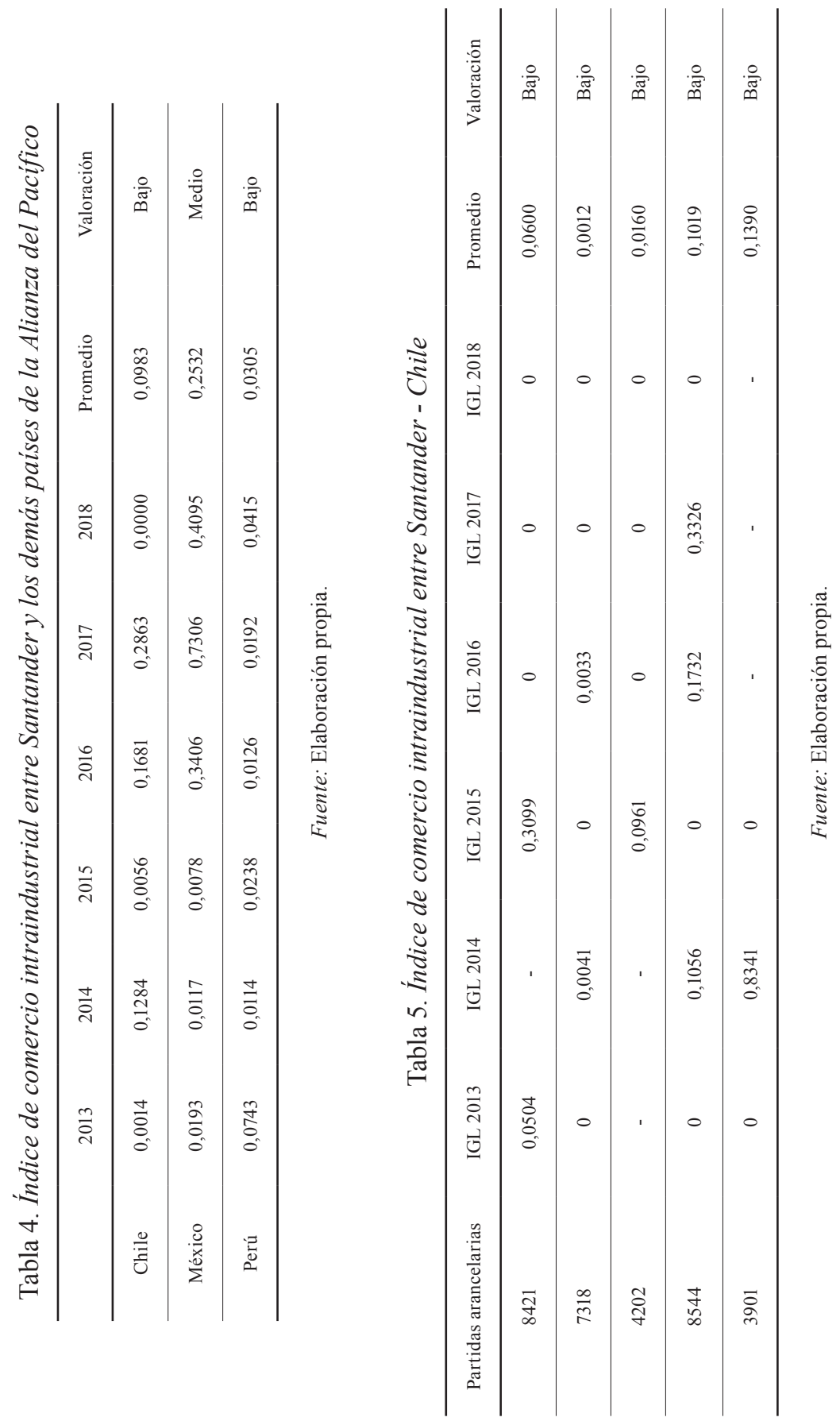


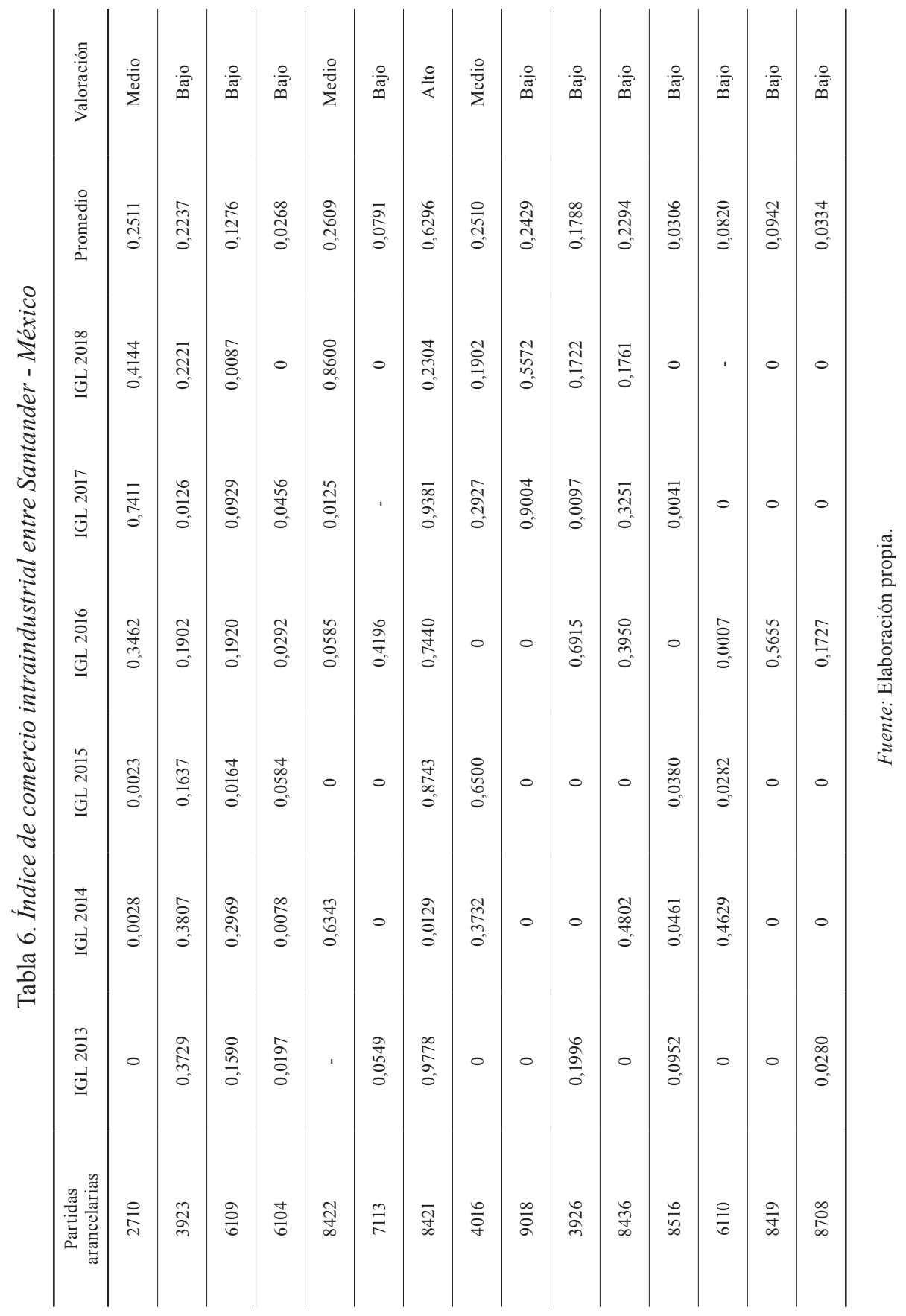




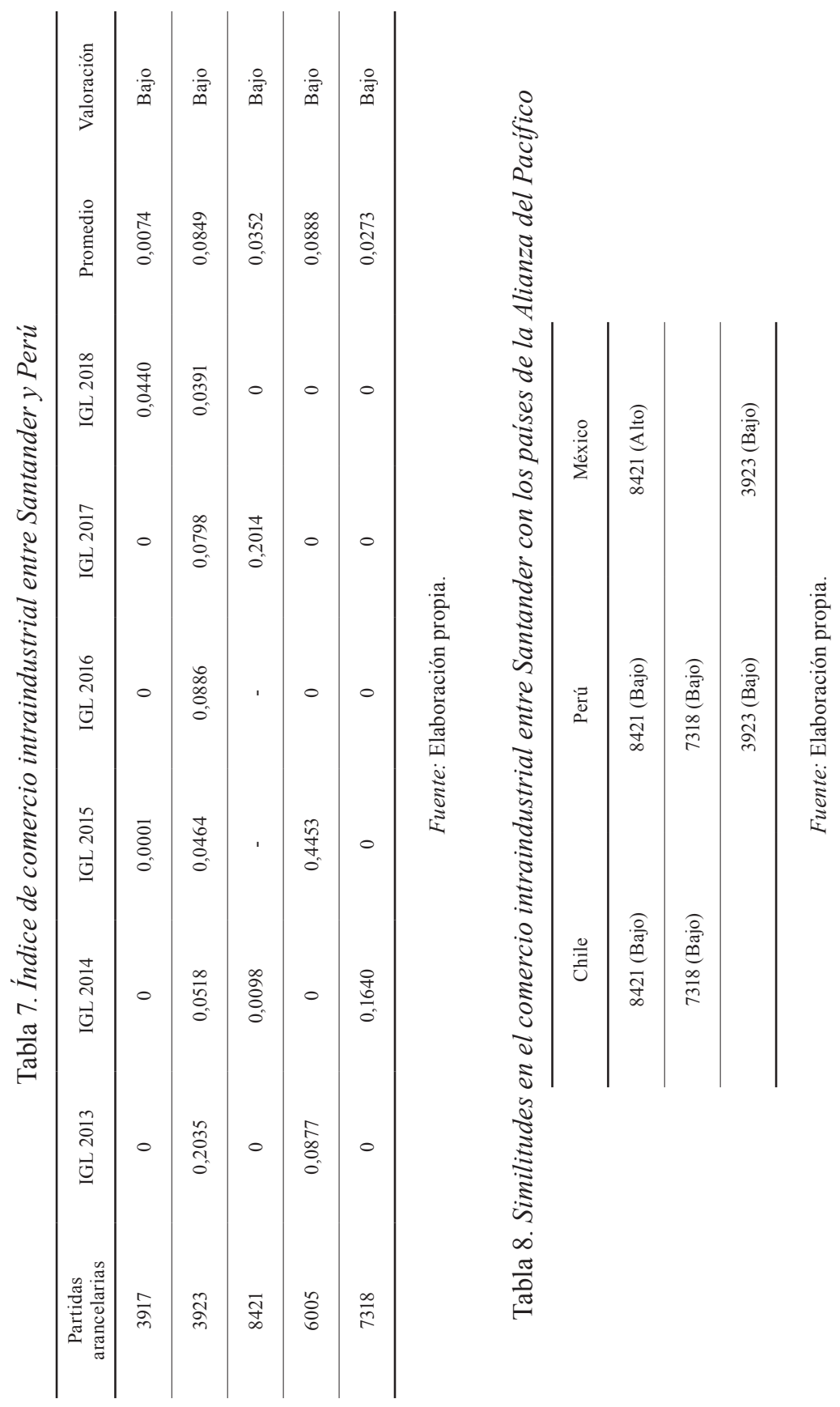




\section{Conclusiones}

Contrario a la disminución de las exportaciones totales de Santander al mundo, entre 2013-2018, se presentaron aumentos en el mismo periodo con cada uno de los mercados de la AP. Los países de la Alianza del Pacífico en su conjunto representaron un $14,31 \%$ de las exportaciones realizadas por el departamento para el 2018 ; mientras que de forma individualizada, el país que presentó mayor participación en las exportaciones santandereanas entre los países de la Alianza en el 2018 fue México con 5,59\%, seguido por Chile con 4,74\% y Perú con 3,96\%.

Teniendo en cuenta los resultados del índice de Grubel y Lloyd para el comercio intraindustrial entre Santander, con respecto a cada uno de los países miembros de la Alianza del Pacífico para el periodo 2013-2018, se evidencia un nivel bajo de comercio intraindustrial, especialmente con Chile y Perú y medio con México. A nivel de partidas arancelarias, en muy pocos casos se presentó comercio intraindustrial medio o alto; en la mayoría de partidas donde se presentó comercio intraindustrial de Santander con Chile, México y Perú, el valor del índice Grubel y Lloyd fue bajo.

Se encontró que Santander exportó en promedio productos de 112 partidas arancelarias a los países de Chile, México y Perú, integrantes junto con Colombia de la Alianza del Pacífico, con valores superiores a 3000 dólares en al menos un año, y de ellas en 25 partidas $(22,3 \%)$ se presentó comercio en doble vía, es decir, comercio intraindustrial. El nivel de comercio intraindustrial en dichas partidas, en promedio, fue predominantemente bajo para todos los años entre 2013-2018.

El comercio intraindustrial entre Santander con respecto a los países de la Alianza del Pacífico es predominantemente bajo durante los años 2013-2018; sin embargo, los flujos comerciales con México evidencian comercio intraindustrial medio entre 2013-2018.

Se destaca que el comportamiento del comercio intraindustrial de Santander con respecto a Chile y México se ha caracterizado por una tendencia decreciente entre 2013-2015, un aumento significativo entre 2016 y 2017 y una disminución en el 2018. En el 2018 Santander no presentó ningún tipo de comercio intraindustrial con Chile.

El comercio intraindustrial de Santander con Perú ha sido fluctuante, solo se encontró comercio en doble vía para cinco partidas arancelarias en el periodo del estudio, y en todos los casos el nivel es bajo a excepción de la partida 6005 con nivel medio para el año 2015.

Se identifica que solo el producto 8421 es común en comercio intraindustrial entre Santander con Chile, México y Perú; con nivel alto para México; mientras que las partidas 3923 son comunes en el comercio entre el departamento, Perú y México y la 7318 con Chile y Perú, con nivel bajo. 
Todo lo anterior indica un bajo comercio intraindustrial del departamento de Santander con los países de la Alianza del Pacífico, hecho que refleja baja similitud en las ofertas exportables y pocas oportunidades para la complementariedad. Además, para el departamento de Santander se aprecian ofertas comerciales diversificadas en cada país de la Alianza, ya que la similitud en los productos en doble vía con cada país es muy baja.

Los países de la Alianza del Pacífico ofrecen oportunidades para las exportaciones de Santander por el crecimiento en la participación y valores exportados a cada uno de estos mercados, además los bajos niveles de comercio intraindustrial se constituyen en un reto hacia la búsqueda de la complementariedad productiva y el establecimiento de alianzas hacia la constitución de cadenas de valor regionales que permitan aprovechar ventajas comparativas de localización y proyección a otros mercados.

\section{Referencias}

Aghlmand, S., Rahimi, B., Farrokh, H., Nabilou, B., y Yusefzadeh, H. (2018). Determinants of Iran's bilateral intra-industry trade in pharmaceutical industry. Irinian Journal of Pharmaceutical Research, 17(2), 822-828.

Arza, C. N., y Almanza, R. C. (2014). Determinantes del comercio intraindustrial entre Colombia y sus principales socios comerciales. Revista de Economía del Caribe (13), 36-61.

Aziz, N., Hossain, B., y Mowlah, I. (2018). Does the quality of political institutions affect intra-industry trade within trade blocs? The ASEAN perspective. Applied Economics, 50(33), 3560-3574. https://doi.org/10.1080/00036846.2018.1430336

Baccini, L., Dur, A., y Elsig, M. (2018). Intra-industry trade, global value chains, and preferential tariff liberalization. International Studies Quarterly, 62(2), 329-340. https://doi.org/10.1093/isq/sqy006

Brakman, S., Garretsen, H., y Van Marrewijk, C. (2009). The new introduction to geographical economics. Cambridge University Press.

Caicedo, C., y Mora, J. J. (2011). Comercio intraindustrial Colombia-Estados Unidos. El caso de los bienes altamente tecnológicos (1995 - 2005). Revista Cuadernos de Economía, 30(54), 83-104.

DIAN (Siex). Sistema estadístico de comercio exterior. Recuperado de http://websiex. dian.gov.co/

Duregger, R. (2019). Comércio intraindustrial: Um exame da indústria automobilística brasileira (2000-2016). (Tesis de maestría). Universidad Estadual Paulista, Araraquara, Brasil. 
Gaya, R., y Michalczewsky, K. (2014). El comercio intrarregional sudamericano: patrón exportador y flujos intraindustriales, pp. 1-102. Banco Interamericano de Desarrollo BID.

Hernández, J. A., y Muñoz, L. G. (2015). Comercio y evolución de la alianza del pacífico. Equidad y Desarrollo, 24, 97-118.

Jiménez, J. (2018). Análisis del comercio intraindustrial entre Colombia y Estados Unidos para el periodo 1991-2015. [Tesis de pregrado]. Universidad del Valle, Cali, Colombia.

Kalim, S. (2018). David Ricardo's comparative advantage and developing countries: myth and reality. International Critical Thought, 8(3), 426-452. https://doi.org/10 $.1080 / 21598282.2018 .1506264$

León, G. P. A., y Dussel, E. (2001). El comercio intraindustrial en México, 19901999. Comercio Exterior, 51(7), 652-664.

López, J. A., y Rodil, O. (2008). Comercio intraindustrial e intrafirma en México en el contexto del proceso de integración de América del Norte (1993-2006). Economía UNAM, 5(13), 86-112.

Lucangeli, J. (2007). La especialización intraindustrial en Mercosur. Comisión Económica para América Latina y el Caribe (CEPAL). Serie Macroeconomía del desarrollo (64), 1-92.

Márquez, A. (2015). Desafíos a enfrentar por el comercio intrarregional en la Alianza del Pacífico. Aldea Mundo, 20(40), 71-84.

Molinari, A., Bembi, M., y de Ángeles, J. Y. (2012). Medición de la integración productiva en el Mercosur: un análisis desde la óptica del comercio intraindustrial y las cadenas de valor. Desarrollo económico, 52(207-208), 511-544.

Montaner, J. M., y Orts, R. V. (1996). Naturaleza y causas del comercio intraindustrial. Ekonomiaz: Revista Vasca de Economía, 36, 78-101.

Moreno, A. I., y Posada, D. H. M. (2006). Definición teórica y medición del comercio intraindustrial. Borradores del CIE, 26, 1-19. Universidad de Antioquia.

Moreno, A. I., y Posada, H. M. (2007). Evolución del comercio intraindustrial entre las regiones colombianas y la Comunidad Andina, 1990-2004: Un análisis comparativo. Lecturas de Economía, 66, 83-118.

Moscoso, F. F., y Vásquez, H. (2005). Determinantes del comercio intra-industrial en el grupo de los tres. Universidad Externado de Colombia. Bogotá, Colombia.

Pick, D. H., Henderson, D. R., Kinsey, J. D., y Sheldon, I. M. (2018). Global markets for processed foods, theoretical and practical issues. New York: Routledge. 
Sotomayor, M. L. (2009). Medición del comercio intraindustrial no maquilador de México. Investigación económica, 68(268), 39-68.

Sotomayor, M. L. (2008). Un estudio sobre el comercio intraindustrial de México en el tratado de libre comercio de América del Norte. (Tesis doctoral). Universidad de Barcelona, Barcelona, España. Recuperado de https://ddd.uab.cat/pub/ tesis/2008/tdx-0401109-162308/mlsylde1.pdf

Villalobos, E. A., y Quiroga, J. C. A. (2017). Cálculo de los determinantes del comercio intraindustrial entre México y Estados Unidos para el periodo de 2005 a 2016. Management Review, 2(1), 1-8.

WISERTrade. World Institute for Strategic Economic Research. Recuperado de http://www.wisertrade.org 